\title{
Avant-propos à Libre échange
}

\section{Luis Araújo}

\section{(2) OpenEdition}

\section{Journals}

Édition électronique

URL : https://journals.openedition.org/cher/418

DOI : $10.4000 /$ cher.418

ISSN : 2803-5992

\section{Éditeur}

Presses universitaires de Strasbourg

\section{Édition imprimée}

Date de publication : 19 novembre 2020

Pagination : 209-212

ISBN : 979-10-344-0071-3

ISSN : 1968-035X

\section{Référence électronique}

Luis Araújo, «Avant-propos à Libre échange », reCHERches [En ligne], 25 | 2020, mis en ligne le 01 octobre 2021, consulté le 17 novembre 2021. URL : http://journals.openedition.org/cher/418 ; DOI : https://doi.org/10.4000/cher.418 


\title{
Avant-propos à Libre échange ${ }^{1}$
}

\author{
Luis Araúso
}

On recourt souvent à la nature humaine pour justifier la volonté de réussite, de puissance et de domination. La compétitivité comme système naturel de l'évolution de l'espèce. Un long parcours nous conduit de la Loi de la jungle au Siècle des Lumières et à ses efforts pour construire une société sur les principes de la raison, de l'égalité et du droit. Dotés de la raison, nous sommes capables de penser l'égalité et la solidarité. Mais «le sommeil de la raison» a créé autant de monstres que l'instinct sauvage. Jusqu'à nos jours ni la politique, ni la religion, ni les arts n'ont réussi à trouver de réponse au paradoxe de pulsions qui nous permettent de progresser tout en nous détruisant. Cette contradiction interne, ne serait-elle pas l'essence même de la nature humaine?

Ainsi en ce début du $\mathrm{xxI}^{\mathrm{e}}$ siècle, nous sommes confrontés à ce croisement: «ange sauvagement humain» selon les mots de Blas de Otero. Pour qu'il y ait progrès, il n'y a d'autre solution que de canaliser les deux forces naturelles capables de nous transformer en êtres sublimes ou en monstres: l'instinct de domination et le corset rationaliste.

L'économicisme, machine à broyer, transforme tout notre environnement: l'espace et le temps, la géographie et le climat, les sociétés et les civilisations, les relations interpersonnelles et la mémoire des peuples. Et, aujourd'hui, même les droits les plus élémentaires à exister et à s'alimenter se réduisent à des chiffres que l'on manipule en fonction de la spéculation criminelle des marchés. Vive le libre échange! Et, sur le chemin, nous piétinons les lambeaux de cette partie de l'humanité, la plus grande, mise en miettes. Vive le libre échange, mais quand le marché menace de s'effondrer il faut tout sacrifier pour injecter des fonds, pour que la machine ne s'arrête pas, même s'il faut pour cela l'alimenter de la chair fraîche de ses victimes. Vive le libre échange, bien que l'adjectif ne s'applique qu'à ceux qui pensent les lois dans le seul but de servir leur pouvoir, et

1 Ce texte reprend en partie l'introduction et quelques-uns des commentaires que Luis Araújo a écrits pour l'édition que le Teatro Español a faite de ce texte: Mercado libre, Madrid, Cuadernos del teatroespañol, 2009. 
certainement pas à ceux qui pourraient déstabiliser notre balance de privilèges. Vive le libre échange tant que nos bénéfices sont garantis.

Et, soudain, le marché libre fait crack comme une bulle de savon et alors on réclame l'intervention de l'état, qui ne peut pas laisser sans protection ses citoyens si respectables et si libres, ceux qui, pendant des années, ont spéculé et se sont remplis les poches sur le dos des gens et de leurs besoins primaires: logement, alimentation, droit au travail, à la santé, à la vie. Marché libre pour les citoyens de classe A, bien sûr. Car si le citoyen de classe B essaie d'entrer dans le jeu, on lui signifiera aussitôt qu'il ne réunit pas les conditions suffisantes pour se retrouver en première division. Il n'a ni les papiers nécessaires, ni suffisamment peu de scrupules, ni le pedigree requis: il n'a ni la bonne couleur de peau, ni la nationalité adéquate, ni l'appui du clan ou de la classe sociale, ni... Comment ose-t-il prétendre avoir le droit d'entrer dans le jeu de la concurrence d'égal à égal?

Voilà le jeu et les valeurs dans lesquels on nous éduque, on nous entraîne, on nous prépare pour la vie. Nous voulons des enfants capables de s'imposer, de triompher, c'est-à-dire d'être compétitifs. Nous les voulons capables de montrer qu'ils ont des tripes pour construire leur avenir dans la jungle de la vie. Car qui n'y réussit pas, est coupable. Peut être un peu faible. En tous les cas incapable. Probablement paresseux, suspect... Peut-être même le soupçonnera-t-on d'être impliqué dans des affaires louches et délictueuses. Car, quelqu'un a t-il encore un doute aujourd'hui : celui qui vaut, vaut?

C'est ainsi que les relations personnelles se transforment peu à peu en relations commerciales. Puisque tout ce que nous faisons, sortir, nous promener, manger, aller faire les courses ou au cinéma avec quelqu'un, deviennent des activités utiles pour les intérêts économiques, professionnels ou d'ascension sociale. Nous trouvons chaque fois moins de temps pour la famille, les amis, les voisins du quartier, les camarades d'études... À toutes les époques, le théâtre a puisé ses thèmes dans cette mine inépuisable que sont les relations de couple, construites en fonction de ce type d'intérêts. Le néolibéralisme transforme aujourd'hui notre vie en une simple activité commerciale. Il envahit la vie privée comme jamais auparavant. Il globalise tout, pas seulement géographiquement (toute la planète est mise en vente), mais surtout dans le sens de cette idéologie, l'économicisme (tout dans la vie est économie). On instaure la liberté du marché comme la seule régulation possible. On subordonne toutes les formes de vie à des vecteurs économiques. On remplace les valeurs éthiques par des valeurs de marché. Tout a un prix. Nous avons tous un prix.

Libre échange est une histoire sur les humiliations auxquelles le marché soumet les personnes transformées en marchandises. Le face à face des deux personnages de la pièce, un avocat et une prostituée immigrée, me semble la meilleure métaphore pour incarner le système: nous sommes dans un état de droit régulé par les lois, mais dans le même temps, une loi non écrite nous dit que tout est marchandise. 
A et B sont vous et moi, chacun d'entre nous. Les relations qu'ils entretiennent ressemblent furieusement aux nôtres... tout est une question de degré...

Jusqu'où peuvent aller les lois du marché hors de toute régulation, lorsque que certains manient en toute liberté les fils des transactions? Tout accord privé entre deux personnes est-il une transaction d'égal à égal, libre et légitime? Tout achat-vente est-il acceptable? Pour qui n'a rien, jusqu'où peut conduire la nécessité de survivre dans un tel monde? Et qui a tout, jusqu'où est-il prêt à aller? Jusqu'où peut conduire la volonté de puissance, le désir de posséder les personnes et les choses? Jusqu'à quels désirs peu avouables peut mener l'insatiable obsession d'obtenir tout ce dont on a envie? Y a t-il une limite au désir humain?

J'ai essayé de montrer, dans Libre échange, que la relation entre deux personnes, réduite à de successives transactions, peut passer par la froide négociation, l'admiration mutuelle, l'éveil de sentiments obscurs, l'affirmation de la différence, le rejet, les liens de dépendance morbide, la répulsion, la domination, la haine et la destruction.

J'ai élaboré les dialogues de manière à ce que les répliques soient des actions et s'enchaînent comme des actions, et j'ai éliminé les didascalies, ou presque. Ce qui m’a conduit à des répliques concentrées et aiguisées à l'extrême et à jouer avec les silences. J'affectionne les silences éloquents. Ils sont eux-mêmes des répliques, des actions. Je les fais varier en intensité: brève pause, pause, silence, long silence, très long silence. Question de rythme, mais plus encore de créativité émotionnelle que j'offre à l'acteur et au metteur en scène. Les silences sont le territoire de l'implicite, ils sont les portes sémantiques qui communiquent avec l'imagination du spectateur.

Jesús Cracio, metteur en scène de la pièce pour le Teatro Español en décembre 2009, a su bien comprendre et exposer le ton et l'esthétique qui convenaient le mieux à mon texte:

Au début du mois de novembre (2008) Paco Pena² me contacte et me propose de réaliser la mise en scène d'une pièce pour la Petite salle du Teatro Español: Libre échange. Il me l'envoie par courriel et dans la nuit même je lis d'une traite le texte. Deux personnages - avec les années, j'aime de plus en plus travailler avec peu d'acteurs, car en trop grand nombre ils vocifèrent beaucoup trop sur scène - : un avocat corrompu et une prostituée immigrée. Et un thème qui m'obsédait alors: la chair et l'âme mis en vente. [...]

Luis Araújo, volontairement, ne donne pas de noms aux personnages: juste A, citoyen et B, sans papiers. Il va droit au but. La pièce organisée en 14 courtes scènes ne nous situe dans aucun espace déterminé, physique ou temporel, elle ne donne aucune indication sur l'état émotionnel des personnages. Pas davantage de précisions concernant l'éclairage et les éléments scénographiques. [...] «A demande à $\mathrm{B}$ de se masturber», et aussitôt ils se lancent dans une disquisition de trois pages sur l'achat-vente, mais à aucun moment il n'est indiqué: «B se

2 Paco Pena est le chef de production du Teatro Español de Madrid. 
masturbe». Je vois deux acteurs qui s'exposent et mettent à nu leurs âmes plus que leurs corps. [...]

Depuis le début je me suis entêté: je voulais une actrice latino-américaine pour le rôle de $\mathrm{B}^{3}$. [...] Je voulais un sentiment et un regard non européens, quelqu'un qui, sans être immigré, ait été arraché à ses racines. C'est ainsi que j’ai choisi Yoima Valdès. [...]

Pour les transitions d'une scène à l'autre, j'ai opté pour le changement de vêtements à la vue des spectateurs. Ce qui a permis de souligner les évolutions internes des personnages, de montrer ce qui se passait entre les scènes et, en même temps, de préparer la charge émotionnelle de l'acteur nécessaire à la scène suivante. ${ }^{4}$

Mercado libre a été porté à la scène grâce à un message de Mario Gas, à l'époque, directeur du Teatro Español de Madrid, intéressé par ce texte présenté au Prix Lope de Vega de la Ville de Madrid et non retenu, et souhaitant connaître le nom de son auteur. Je le remercie donc d'avoir fait confiance dès le premier moment à cette pièce sans même savoir qui en était l'auteur.

3 Dans sa première version du texte, Luis Araújo avait fait de B une Mexicaine.

4 Déclarations dans l'édition de Mercado Libre (Araujo 2009), extraits du texte de Jesús Cracio, p. 119-132, ici p. 120-121, 122, 127 et 130. 